\title{
Bauphysik und bauliche Maßnahmen
}

\author{
Daniel Büth, Wolfgang Meißner
}

Die RFID-Technologie ist eine bereits seit vielen Jahren genutzte Technologie, welche in einer Vielzahl von Anwendungen fester Bestandteil unterschiedlicher Prozesse ist. Grundsätzlich arbeiten RFID-Geräte auf dem aktuellen Stand der Technik bei fachgerechter Installation zuverlässig und störsicher. Trotzdem sind dies Funkanlagen, welche durch andere Sender, wechselnde Umgebungsbedingungen oder andere Störquellen beeinflusst werden können. Aus den Erfahrungen, welche in weit über 3000 verschiedenen Installationen in unterschiedlichen Bibliotheken und einer Vielzahl von Installationen in anderen Branchen gesammelt wurden, lassen sich verschiedene Richtlinien für die Installation von RFID-Systemen ableiten. Die folgenden Kapitel liefern einen Überblick über verschiedene Anwendungen der RFID-Technologie in Bibliotheken und stellen hilfreiche Hinweise zur Vermeidung von Fehlern und Störungen zur Verfügung. Dabei wird ein besonderes Augenmerk auf die Installation von Gates zur Warensicherung gelegt. Diese sind die leistungsstärksten Komponenten einer Installation und damit verbunden auch diejenigen, welche zum Einen selbst sehr störanfällig sind, zum Anderen aber auch mit anderen Geräten interferieren können.

\section{Einleitung}

Die RFID-Technik wird bereits seit vielen Jahren erfolgreich in verschiedenen Bereichen von Industrie, Handel, Verkehr und Logistik eingesetzt. Dabei können mit Hilfe der Transponder und Lesegeräte verschiedene Prozesse zuverlässiger gestaltet und optimiert werden.

Auch im Bereich von Bibliotheken hat sich die RFID-Technik weitgehend durchgesetzt. Hier ergeben sich eine Vielzahl verschiedener Vorteile für Besucher und Betreiber der Bibliotheken. So ermöglicht die RFID-Technologie beispielsweise eine einfache und bequeme Selbstverbuchung der Medien durch den Besucher selbst. Ebenso kann eine Ausleihe und Rückgabe 24 Stunden, 7 Tage die Woche erfolgen. Durch RFID-Gates an den Ein- und Ausgängen der Bibliothek kann eine Diebstahlsicherung der Medien gewährleistet werden. Verschiedene Prozesse, wie die Sortierung, Katalogisierung oder Inventarisierung können automatisch gestaltet werden, wodurch eine erhebliche Zeit- und Kostenersparnis erzielt werden kann.

Die technischen Prozesse sind weitgehend bekannt und werden ständig verbessert. Dabei profitiert man besonders von der Tatsache, dass mittlerweile RFID-Geräte und Transponder nach dem Standard ISO/IEC 15693 und der Frequenz 13.56MHz weltweit in über 3000 Bibliotheken eingesetzt werden. Trotzdem können verschiedene bauliche Gegebenheiten wie ungünstige Positionierung der Leser und Antennen sowie Störungen die Erkennungseigenschaften beeinflussen. 
Nach dem erfolgreichen Einzug der HF-Technologie beginnen nun erste Pilotprojekte mit der Einführung der UHF-Technologie (860-960 MHz) in den Bibliothekssektor. Dabei treten zum Einen ähnliche Problemstellung wie im HF-Bereich auf, zum Anderen sind aber auch vollkommen neue Herausforderungen zu bewältigen.

Im Folgenden wird deshalb eine separate Betrachtung der beiden Frequenzbänder durchgeführt. Dabei sind verschiedenen Leseszenarien, welche in einer Bibliothek Anwendung finden können, einzeln zu berücksichtigen. Mögliche Lesepunkte, welche im Folgenden näher betrachtet werden, sind:

- RFID-Gates zur Artikelsicherung an Ein- und Ausgängen

- Selbstverbuchungsautomaten zur Ausleihe durch den Besucher selbst

- Mitarbeiterplätze

- Rückgabeautomaten

- Automatische Sortieranlagen

Die hier beschriebenen Hinweise sollen helfen, bereits bei der Planung die Position und Ausstattung der Bibliothek mit RFID-Geräten zu optimieren.

\section{HF-Antennen-Gates zur Diebstahlsicherung}

RFID-Antennen-Gates werden zur Diebstahlsicherung an allen Ein- und Ausgängen der Bibliotheken installiert. Diese Antennen-Gates können als Einzeldurchgang "SingleGate« (zwei Antennen), »Double-Gate« (drei Antennen) oder mit mehreren Antennen als »Multiple-Gate« aufgebaut werden.

Gute Leseraten können nur dann erreicht werden, wenn die Antennen im richtigen Abstand zu größeren Metallteilen und möglichen Störquellen wie zum Beispiel Energiekabel im Boden oder Wänden montiert sind.

Typische Fehlerquellen, die zu einem unzuverlässigen Betrieb der Geräte führen können, sind:

- Zu große Durchgangsbreiten der Antennen-Gates

- Geländer, Regale oder Tische mit Metallrahmen in unmittelbarer Nähe der Antennen

- Metallträger oder andere große Metallteile in unmittelbarer Nähe der Antennen (Wänden)

- Gegenseitige Beeinflussung der verschiedenen RFID Installationen

- Energiekabel mit hohen Strömen oder andere magnetische Störer in unmittelbarer Nähe der Antennen 


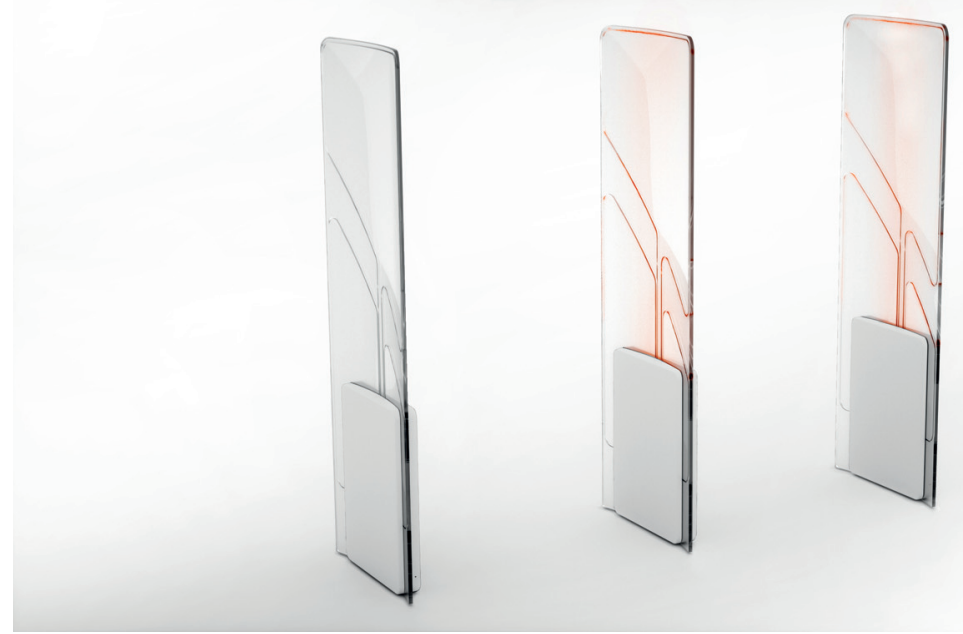

Abb. 1: HF-Crystal-Gate der FEIG ELECTRONIC GmbH mit Alarmleuchte

Viele Antennen besitzen keinen hundertprozentig, dreidimensionalen Erfassungsbereich. Daraus ergeben sich einige Leselöscher im Durchgang in speziellen Ausrichtungen der Transponder. Um ein Höchstmaß an Sicherheit zu gewährleisten, ist es jedoch unabdingbar, dass eine dreidimensionale Erfassung des Transponders innerhalb des Gates möglich ist. Dies bedeutet: unabhängig davon, in welcher Position der Transponder durch das Gate hindurchbewegt wird, auf die komplette Gatebreite gesehen gibt es immer mindestens eine Position in der der Transponder sicher erkannt werden kann. Um eine dreidimensionale Erfassung sicher zu gewährleisten, sollte die lichte Durchgangsbreite zwischen den Antennen $90 \mathrm{~cm}-105 \mathrm{~cm}$ nicht überschreiten. Kommen Spezialantennen zum Einsatz, so können Durchgangsbreiten von bis zu $130 \mathrm{~cm}$ realisiert werden. Ist eine eindimensionale Erfassung des Transponders ausreichend, so darf die Durchgangsbreite bis zu $160 \mathrm{~cm}$ betragen.

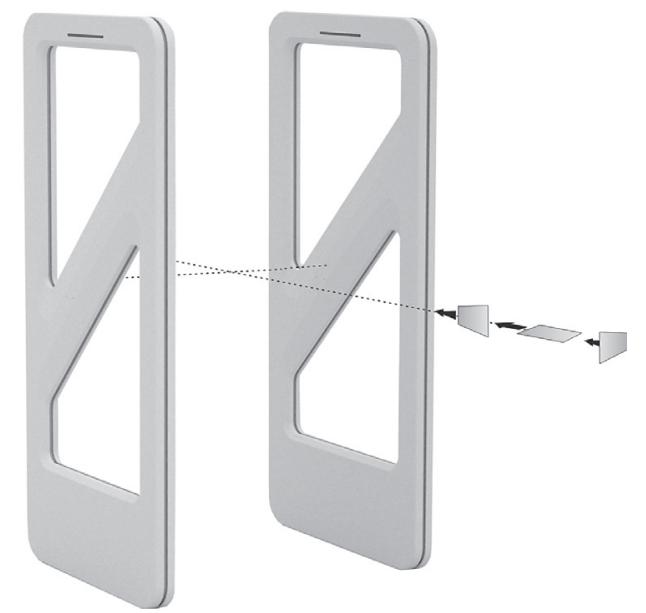

Abb. 2: dreidimensionale Erfassung eines Transponders 
Ein weiteres Problem können Metallteile in der Nähe der Antennen darstellen. Dies betrifft beispielsweise Geländer, Regale oder Tische mit Metallrahmen. Ebenso können dies fest installierte Metallteile wie Rolltreppen oder Fahrstühle und baulich bedingte Metallteile wie Stahlträger oder eine Deckenbewehrung sein. Metall und andere leitende Materialien kann ein magnetisches Feld nicht durchdringen. Dadurch werden der Feldlinienverlauf und die Induktivität der Antenne verändert. Dies resultiert in einer starken Beeinflussung des Systems. Weiterhin wird das Feld durch die Gegeninduktivität bzw. die Wirbelströme im Metall geschwächt. Der Einfluss von Metall auf die Performance / Lesereichweite eines RFID-Systems ist in Abbildung 3 zu sehen.

In dem Bild wird von einer Leser-Antennen-Konstellation ausgegangen, welche unter Idealbedingungen eine maximale Lesereichweite von $80 \mathrm{~cm}$ erzielen kann. Werden Metallteile in die Nähe der Antenne gebracht, so bricht die Leseperformance drastisch ein. Ein größeres Metallteil in 10cm Entfernung von der Antenne bewirkt, dass sich die Lesereichweite halbiert. Bei einer Entfernung von $25 \mathrm{~cm}$ zwischen Metall und Antenne beträgt die Lesereichweite noch 75 \% derer unter Idealbedingungen. Der störende Einfluss von Metall auf die Induktivität einer Antenne kann durch einen erneuten Abgleich der Antennenschleife kompensiert werden. Damit wird die Antenne auf die speziellen Umgebungseinflüsse abgestimmt. Zwar kann damit die Performance des Systems um einiges verbessert werden, jedoch wird es nicht möglich sein den störenden Einfluss von Metall in kurzer Distanz zur Antenne vollständig zu eliminieren. Nach einem erfolgten Antennenabgleich kann ein Transponder über $65 \mathrm{~cm}$ gelesen werden, wenn sich die störenden Metallteile nur $10 \mathrm{~cm}$ entfernt von der Antenne befinden. Bei einer Entfernung von $25 \mathrm{~cm}$ zwischen Antenne und Metall ist nur noch eine geringe Beeinflussung messbar. Hier kann eine Lesung bereits über eine Entfernung von $75 \mathrm{~cm}$ erfolgen.

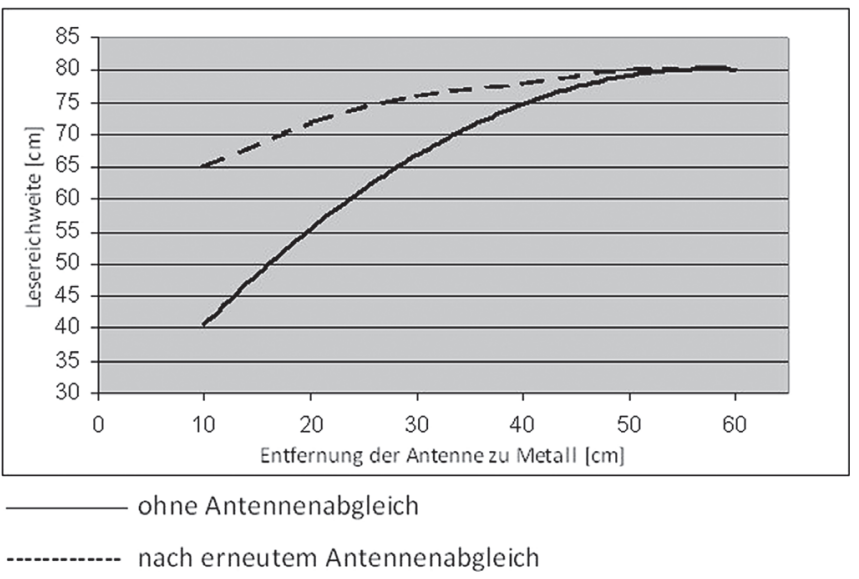

Abb. 3: Einfluss von Metall auf die Performance eines RFID-Systems

Grundsätzlich gilt, dass eine Antenne mindestens in einem Abstand von $50 \mathrm{~cm}$ zu größeren Metallteilen aufgestellt werden sollte, damit eine Beeinflussung des Leseverhaltens vermieden werden kann. Sind Abweichungen von der bestmöglichen Performance des Systems in der Applikation akzeptabel, so sollte dennoch ein Mindestabstand von $20 \mathrm{~cm}$ nicht unterschritten werden. 
Von entscheidender Bedeutung für die Performance eines RFID-Gates ist auch die gegenseitige Beeinflussung verschiedener RFID-Leser untereinander. Hier gilt es folgende Szenarien zu betrachten:

- Lichter Abstand zweier Antennen mit unabhängigen Lesern zueinander

- Lichter Abstand zweier Antennen mit einem gemeinsamen Leser zueinander

- Lichter Abstand einer Antenne zu Selbstverbuchungsautomaten, Mitarbeiterplätzen, Rückgabestationen und Sortieranlagen

Werden zwei Antennen mit zwei unabhängigen Lesern in einer Applikation betrieben (z. B. getrennter Ein- und Ausgang), so sollte ein lichter Mindestabstand von $8 \mathrm{~m}$ zwischen beiden Standorten eingehalten werden. Anderenfalls kann es zu einer Interferenz zwischen beiden Systemen kommen, wodurch eine Identifikation von Transpondern nahezu unmöglich wird. Dieser Abstand kann durch zwei verschiedene Maßnahmen bis auf $1 \mathrm{~m}$ reduziert werden. Hier bietet sich entweder die Möglichkeit beide Leser miteinander zu synchronisieren (Readersynchronisation) oder ein Multiplexen der Leser (Readermultiplexing) durchzuführen. Bei beiden Maßnahmen ist eine zusätzliche Verkabelung erforderlich, welche die beiden Leser miteinander verbindet.

Bei der Readersynchronisation stellt ein erster Leser den Master dar. Zu einem bestimmten Zeitpunkt sendet der Master einen Triggerpuls über die Steuerleitung an die benachbarten Leser. Dieser Puls stellt das Startsignal für die RF-Kommunikation dar. Alle Leser beginnen daraufhin gleichzeitig mit dieser.

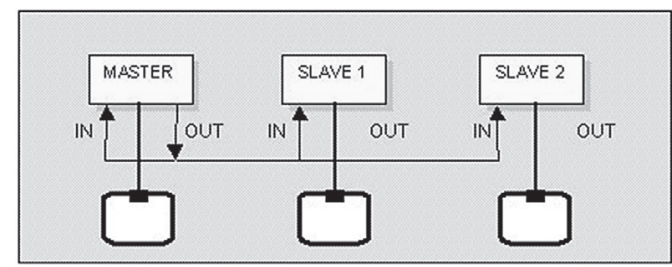

Abb. 4: Prinzip der Readersynchronisation

Im Falle des Readermultiplexings stellt ebenfalls ein erster Leser den Master dar. Dieser ist als erstes berechtigt eine RF-Kommunikation durchzuführen. Hat er diese beendet, so sendet er einen kurzen Puls an einen benachbarten Leser. Daraufhin ist dieser berechtigt eine Kommunikation zu beginnen. Nachdem er diese beendet hat, sendet er wiederum einen Triggerpuls an den nächsten Leser. Beim Readermultiplexing handelt es sich somit um eine Reihenschaltung von Lesern.

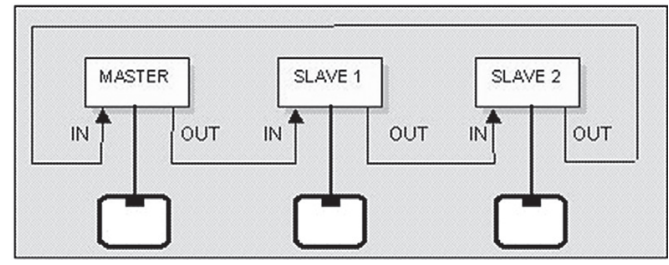

Abb. 5: Prinzip des Readermultiplexing 
Werden mehrere Gates von einem Leser aus angesteuert, so ist eine maximale Kabellänge von $6 \mathrm{~m}$ zulässig. Werden größere Kabellängen benötigt, so sind Performanceverluste mit einzukalkulieren. Bei einer Kabellänge von 11m ist mit etwa 6-10\% Leistungsverlusten zu rechnen.

Der Vorteil beim Betrieb mehrerer Antennen an einem Leser besteht darin, dass die Antennen über einen Multiplexer angesteuert werden können. Dies hilft zum Einen die Kosten für Leser zu senken, zum Anderen kann damit auf zusätzliche Synchronisationsmaßnahmen verzichtet werden.

Ebenfalls kann es zu einer Beeinflussung zwischen RFID-Antennen und Selbstverbuchungsautomaten, Mitarbeiterplätzen, Rückgabestationen und Sortieranlagen kommen. Hier gilt zu berücksichtigen, dass durch zusätzliche Abschirmmaßnahmen Mindestabstände erheblich reduziert werden können. Ohne eine Schirmung sollte ein Mindestabstand von $5 \mathrm{~m}$ zu einem Selbstverbuchungsautomaten nicht unterschritten werden. Mit einer zusätzlichen Schirmung kann dieser auf $2 \mathrm{~m}$ reduziert werden.

Der Mindestabstand zu Rückgabestationen und Sortieranlagen ist immer abhängig von der Sendeleistung, der Größe der Antenne, sowie der Schirmung. Gewöhnlich ist hier mit einem Abstand zwischen 1m und 10m zu rechnen.

Eine weitere mögliche Fehlerquelle, welche die Performance eines Gates zur Warensicherung erheblich beeinflussen kann, stellt eine unsaubere Spannungsversorgung dar. Dies kann beispielsweise durch andere elektronische Geräte hervorgerufen werden, welche am gleichen Stromkreis angeschlossen sind. Hier ist es ratsam einen eigenen Stromkreis für den Betrieb der Gates vorzusehen. Falls dennoch weitere Störungen in der Spannungsversorgung auftreten, kann ein zusätzlicher EMV Filter vorgeschaltet werden.

Problematisch sind ebenfalls Energiekabel, Computermonitore und große Leuchtstoffröhren, welche in der Nähe der Antennen verlaufen oder montiert sind. Zu Energiekabeln ist unbedingt ein Mindestabstand von $1 \mathrm{~m}$ in alle Richtungen einzuhalten. Bei Computermonitoren (CRT-Röhre) sollte ein Abstand von $60 \mathrm{~cm}$ eingehalten werden. $\mathrm{Zu}$ großen Leuchtstoffröhren und Leuchtreklamen gilt es einen Abstand von 2m einzuhalten.

\section{UHF-Antennen-Gates zur Diebstahlsicherung}

Typische Anwendungen für UHF-Antennen-Gates sind auf Grund der großen Lesereichweite, welche mit einem solchen System erzielt werden kann, bisher überwiegend im Bereich der Logistik zu finden. Hier werden UHF-Gates gewöhnlich zur Kontrolle des Wareneingangs und Warenausgangs in Verteilzentren und Märkten verwendet. Diese sind in der Regel recht großzügig und robust konstruiert und daher für einen Einsatz in Bibliotheken, welche hohe Anforderungen an Architektur und Design stellen, nicht geeignet. Dennoch kann von denen im Logistiksektor gewonnenen Erfahrungen in dieser sehr frühen Phase der UHF-Technologie im Bibliothekswesen profitiert werden. 


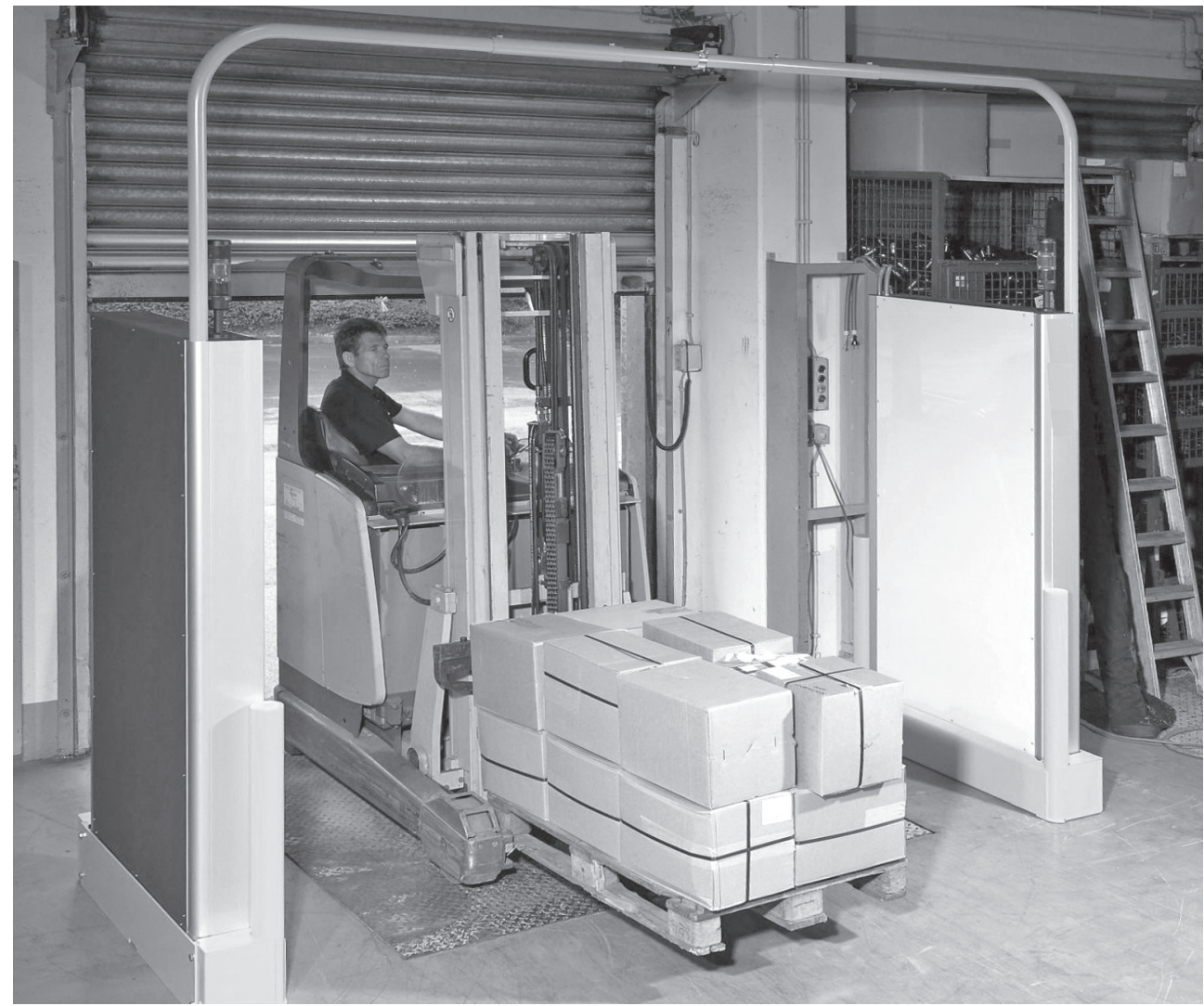

Abb. 6: UHF-Portal im Logistikbereich @Bühnenbau Schnakenberg GmbH \& Co. KG

Im Wesentlichen besteht ein UHF-Gate aus einem Long Range Leser und mehreren Antennen. Diese sind erforderlich um eine lückenlose, dreidimensionale Erfassung innerhalb des Gates sicher zu stellen. Mit nur einer angeschlossenen Antenne kann lediglich eine zweidimensionale Erfassung ermöglicht werden. Die Antennen werden mittels eines Koaxialkabels an den Leser angeschlossen und an beiden Seiten, in den oberen Ecken oder oberhalb des Kontrollpunktes montiert. Auf Grund des begrenzten Öffnungswinkels einer UHF-Antenne (in der Regel ca. $65^{\circ}$ ) ist dabei zu beachten, dass die gesamte Durchgangsfläche erst in einem bestimmten Abstand zur Antenne abgedeckt werden kann. Zwischen den beiden Antennen treten in kurzer Distanz Leselöcher auf, in denen Transponder nicht erkannt werden können.

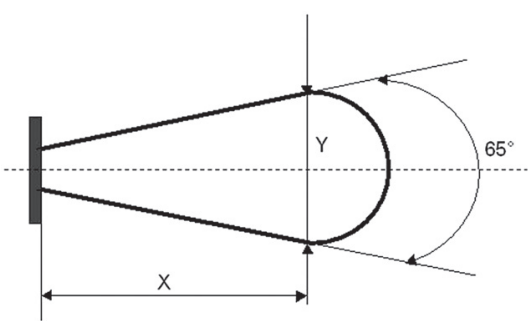

Abb. 7: Öffnungswinkel einer UHF-Antenne 
Deshalb ist bei der Planung der Gates an den Ein- und Ausgängen unter allen Umständen zu beachten, dass die Antennen soweit zurückgesetzt montiert werden, dass im Durchgangsbereich eine lückenlose Erfassung möglich ist. Da nicht permanent Personen durch jedes Gate hindurch gehen, empfiehlt es sich die einzelnen Lesestationen zu triggern. Dazu eignen sich Signalgeber jeder Art, wie z. B. Bewegungsmelder oder Lichtschranken. Alle gängigen UHF-Long-Range-Leser verfügen über mindestens einen digitalen Eingang, auf dem Triggersignale empfangen und zur Steuerung des Lesers verwendet werden können.

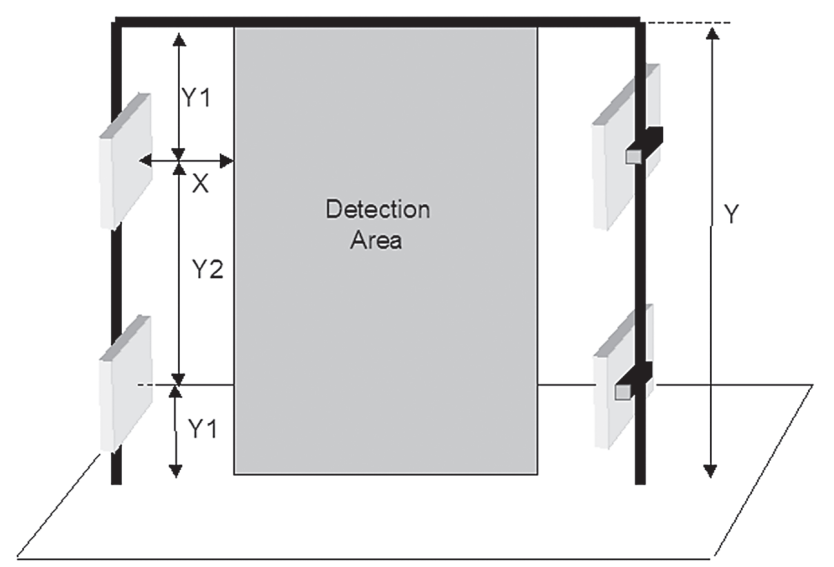

Abb. 8: Typische Anordnung von Antennen in einem UHF-Gate

Der Vorteil der Triggerung besteht dabei darin, dass das RF-Feld des Lesers nur dann eingeschaltet ist, wenn dies unbedingt erforderlich ist. Dies trägt zum Einen dazu bei, den Traffic und mögliche Störungen auf der Luftschnittstelle zu reduzieren, zum Anderen wird durch die Triggerung der Energieverbrauch gesenkt, da im »Standby« die Stromaufnahme des Systems deutlich geringer ist.

Nach dem Standard EN 302208 stehen vier unterschiedliche Sendekanäle mit einer Kanalbandbreite von $200 \mathrm{kHz}$ zur Verfügung. Zwischen den einzelnen Sendekanälen ist ein Schutzband von $600 \mathrm{kHz}$ angesiedelt. Das Kommunikationsverfahren ist so ausgewählt, dass eine beliebige Anzahl von Lesern auf der gleichen Frequenz betrieben werden kann. Dies wird ermöglicht durch die Tatsache, dass die Transponderantwort spektral von den Signalen der Leser getrennt wird und in der Mitte des $600 \mathrm{kHz}$ breiten Schutzbandes angeordnet wird. Dadurch wird eine Überlagerung der Transponderantwort durch andere aktive Leser vermieden.

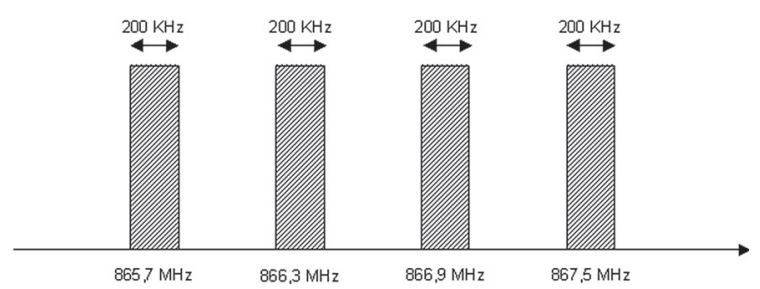

Abb. 9: 4-Kanalplan nach ETSI EN 302208 
Ein typisches UHF-Antennenfeld ist in Abb.10 zu sehen. Dabei fällt auf, dass in einem bestimmten Bereich vor der Antenne ein geschlossenes, homogenes Feld existiert, in welchem ein Transponder sicher erkannt werden kann. Mit zunehmendem Abstand von der Antenne treten erste Leselöcher auf, in denen die Feldstärke nicht ausreichend groß ist, um einen Transponder zu aktivieren. Je weiter man sich von der Antenne entfernt, umso größer und zahlreicher werden diese Leselöcher, bis schließlich nur noch einige wenige Bereiche existieren, in denen der Transponder gelesen werden kann.

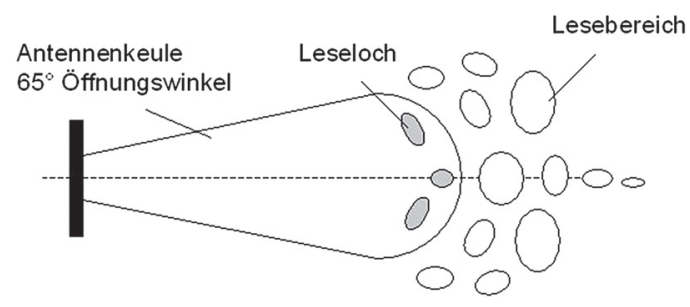

Abb. 10: Typisches UHF-Antennenfeld

Durch Reflektionen kann es sowohl zu einem gehäuften Auftreten von Leselöchern, als auch zu Überreichweiten kommen. Gewöhnlich treten beide Phänomene gleichzeitig auf. Reflexionen entstehen an allen Gegenständen im Bereich eines Gates. Wichtig ist zu beachten, dass durch jede Änderung innerhalb eines UHF-Feldes die Eigenschaften der Applikation verändert werden. Bewegt sich beispielsweise eine Person durch ein Gate, so verändert sich das Feld mit jedem Schritt. Es kommt zu einer ständigen Verschiebung von Leselöchern und Lesebereichen. Kommt es zu einer Überlagerung von Wellen mit um $180^{\circ}$ verschobener Phase, so kommt es zur Auslöschung des Signals und es entsteht ein Leseloch. Überlagern sich Wellen mit gleicher Phase, so kommt es zu einer Verstärkung des Signals, so dass erhebliche Überreichweiten erzielt werden können. Besonders begünstigt werden Reflektionen durch Metall. An metallischen Oberflächen entsteht eine Totalreflektion. Neben dieser Eigenschaft können ebenfalls Wellen auf der Oberfläche eines Metalls weitergeleitet werden. Durch die Weiterleitung von Wellen entlang der Oberfläche kann es vorkommen, dass Transponder, welche sich in großer Entfernung zur Antenne befinden, ebenfalls noch detektiert werden. Dabei können Entfernungen von bis zu 50m überbrückt werden. Typischerweise treten solche Phänomene baulich bedingt auf. Die Wellen werden entlang von Stahlträgern oder Rohren (Heizung, Wasser, etc.) weitergeleitet.

Sind zwei benachbarte UHF-Portale zu dicht aneinander installiert, so besteht die Möglichkeit, dass bedingt durch Reflexionen oder die Weiterleitung von Wellen entlang einer metallenen Oberfläche, Transponder in benachbarten Gates ebenfalls erfasst werden und somit keine eindeutige Zuordnung mehr erfolgen kann. Besonders problematisch ist dies, wenn Ein- und Ausgang dicht nebeneinander liegen.

Während bei Anwendungen im HF-Band (13,56 MHz) benachbarte Gates durch geeignete Synchronisationsmaßnahmen störungsfrei parallel betrieben werden können, sind bei Gateanwendungen im UHF-Bereich verschiedene Szenarien zu unterscheiden. Sind zwei Antennen-Gates mit geringer Durchgangsbreite und minimalem Abstand zueinander 
installiert, so dass durch die beiden Gates eine maximale Distanz von $5 \mathrm{~m}$ abgedeckt wird, dann ist zwischen den beiden Portalen unbedingt eine geeignete Schirmung zu installieren. Dies ist erforderlich, da eine Lesung von Transpondern, welche sich in dem benachbarten Durchgang befinden, erfolgen kann. Ebenso ist es möglich, dass es durch die Einkopplung von Signalen in die Antennen des benachbarten Tores zu einer Übersteuerung des Empfängers des Lesers im Nachbartor kommen kann. Ebenso kann dadurch der Transponder empfindlich gestört werden, so dass dieser keine klaren Informationen empfängt und nicht antwortet. Wird mit zwei Portalen eine Strecke von $6-8 \mathrm{~m}$ abgedeckt, so ist es von der Position der Antennen abhängig, ob eine zusätzliche Trennung der beiden Tore vorgenommen werden muss. Diese ist dann erforderlich, wenn Antennen parallel zueinander montiert sind und direkt ineinander koppeln können. Wird eine Distanz von mehr als $8 \mathrm{~m}$ mit den beiden Toren abgedeckt, so kann auf eine zusätzliche Schirmung verzichtet werden, da bedingt durch die Freiraumdämpfung die abgestrahlten Signale so stark gedämpft werden, dass es nicht mehr zu einer Übersteuerung benachbarter Geräte kommen kann.

Als Abschirmmaterialien zur Trennung der beiden Gates eignen sich Metallplatten oder Absorbermatten. Einfache Metallplatten besitzen den Vorteil, dass sie preiswert sind. Allerdings werden durch zusätzliche metallische Gegenstände im Bereich eines Gates die Anzahl der Reflektionen erhöht, durch welche zusätzliche Leselöcher entstehen und die Performance des Gates verringert wird. Eine lückenlose Erfassung im Bereich innerhalb des Gates kann in diesem Fall nicht sicher gewährleistet werden. Diesen Nachteil besitzen spezielle Matten, welche aus einem absorbierenden Material hergestellt werden, nicht. Hier werden nahezu alle auf die Oberfläche auffallenden Wellen absorbiert. Problematisch ist bei diesen allerdings die Tatsache, dass sie sehr kostenintensiv sind, wodurch der Preis für eine Installation schnell ansteigt.

Befinden sich mit einem Transponder versehene Artikel in der Nähe des Gates oder werden mit einem Transponder gekennzeichnete Bücher oder Medien durch eine Person vor dem Gate vorbei bewegt, kann es, bedingt durch Reflektionen und Überreichweiten, gehäuft zu Fehlalarmen kommen. Deshalb sollte beachtet werden, dass in einem Bereich von mindestens $8 \mathrm{~m}$ vor und hinter dem Gate keine Bücher oder Medien gelagert oder bewegt werden.

Problematisch ist ebenfalls, dass durch die große Reichweite eines UHF-Systems eine Vereinzelung von Personen an Ein- und Ausgängen nahezu unmöglich ist. Dies gestaltet sich dann als problematisch, wenn ein nicht verbuchtes Buch oder Medium in einer größeren Gruppe von Personen durch das Gate getragen wird. Hier kann der Täter nicht eindeutig identifiziert werden und die gesamte Gruppe steht zunächst unter Generalverdacht. Tritt dies entweder durch tatsächlichen Diebstahl oder Fehlalarm gehäuft auf, so könnte dies schnell den Unmut der Besucher nach sich ziehen.

Neben Metall stellen Flüssigkeiten ein weiteres kritisches Element dar. Flüssigkeiten führen zugleich zu Reflektionen und Absorptionen. Wellen werden an der Oberfläche von Flüssigkeiten gebrochen und teilweise reflektiert. Der nicht reflektierte Teil wird von der Flüssigkeit absorbiert. Eine Durchdringung der Flüssigkeit ist nahezu ausgeschlossen. 
Dies kann ebenfalls zu Problemen bei der Warensicherung führen. Da der menschliche Körper zu über 70\% aus Wasser besteht, ist er hervorragend geeignet Transponder abzudecken. Werden Bücher in der Mitte einer größeren Gruppe von Personen durch ein Gate bewegt oder unter dem Arm getragen, so wird eine erfolgreiche Identifikation deutlich erschwert und je nach Grad der Abdeckung des Transponders sogar unmöglich.

\section{Selbstverbuchungsplätze. Mitarbeiterplätze, Rückgabeautomaten und Sortieranlagen mit HF-Technologie}

In modernen Selbstverbuchungseinheiten und Mitarbeiterplätzen werden gewöhnlich Mid-Range-Leser und Antennen in der Größenordnung eines DIN A4 Blattes eingesetzt. Mit einer vom Leser abgegebenen Sendeleistung von 1 Watt und einem typischen Buchtransponder (Größe: $45 \mathrm{~mm}$ x $76 \mathrm{~mm}$ ) ist eine Lesereichweite von $30-40 \mathrm{~cm}$ bei paralleler Labelorientierung zur Antenne möglich.

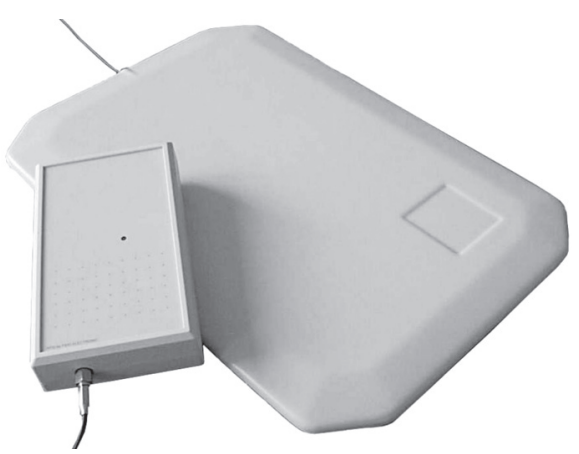

Abb. 11: Leser und Padantenne für Selbstverbuchungsautomaten und Mitarbeiterplätze

Solche Systeme werden typischerweise in der Nähe von Ein- und Ausgängen montiert. Dadurch können diese Stationen relativ Nahe an die Gates zur Artikelsicherung heranrücken. Um längere Wartezeiten zu vermeiden, werden oftmals mehrere Einheiten nebeneinander aufgestellt. Aus diesem Grunde ist es erforderlich zweierlei Mindestabstände näher zu betrachten.

- Mindestabstand zu Antennen der Gates

- Mindestabstand zu benachbarten Selbstverbuchungsstationen

$\mathrm{Zu}$ benachbarten Gate-Antennen sollte der Abstand mindestens $5-7 \mathrm{~m}$ betragen. Dies ist abhängig von den verwendeten Antennen. Da die Leser in Selbstverbuchungsplätzen, Rückgabeautomaten oder Mitarbeiterplätzen mit wesentlich geringerer Leistung operieren, als dies bei Gates der Fall ist, sollte lediglich ein Abstand von 2 -3m zwischen zwei benachbarten Stationen eingehalten werden.

Durch zusätzliche Abschirmmaßnahmen können die angegebenen Mindestabstände weiter reduziert werden. Einen positiven Nebeneffekt stellt die Tatsache dar, dass hierdurch ungewollte Lesungen von Transpondern im Bereich außerhalb der Antenne verringert werden. Prinzipiell sind zwei unterschiedliche Ausführungen der Schirmung denkbar. 
- Abschirmung der Antenne an 5 Seiten mit Hilfe einer Wanne aus Metall.

- Einschränkung des Erfassungsbereichs durch eine flach aufliegende Metallplatte mit Antennenaussparung.

Die Abschirmung der Antenne mit einer Wanne kann aus Metallblech oder Metallfolie hergestellt werden. Dabei sollte die Wanne so ausgelegt werden, dass parallel zur Antennenfläche ein Mindestabstand von $10 \mathrm{~cm}$ gewährleistet ist. Der seitliche Abstand der Wanne zur Antenne sollte mindestens $6 \mathrm{~cm}$ betragen.

Die Metallplatte zur Einschränkung des Erfassungsbereichs sollte am Rande der Antenne verlaufen. Der seitliche Mindestabstand der Schirmung zur Antenne sollte 2 -3cm betragen.

In RFID-Rückgabeautomaten sind oft Antennen der Größe $20 \mathrm{~cm}$ x $20 \mathrm{~cm}$ bis $30 \mathrm{~cm} \mathrm{x}$ $30 \mathrm{~cm}$ installiert. Die Medien liegen hier horizontal im Automaten. Dadurch ergibt sich in der Regel eine eindimensionale Erfassung. Oft befinden sind diese Automaten in einem geschlossen Metallgehäuse. Hier ist es besonders wichtig die notwendige Reichweite und Lesegeschwindigkeit zu kennen, damit das System passend ausgelegt werden kann und der störende Einfluss des Metalls eine erfolgreiche Umsetzung des Szenarios nicht verhindert.

Auch in Sortieranlagen liegen die Bücher/Medien oft auf einem Förderband oder anderen kleinen Transportanlagen. Hier ist ebenfalls nur eine eindimensionale Erfassung erforderlich.

Typische Probleme, welche in Bezug auf Rückgabeautomaten oder Sortieranlagen auftreten können, sind:

- Störungen in der Antenne

- Unzureichende Lesereichweite der Medien (CD, DVD,...)

- Zu geringe Lesegeschwindigkeit des RFID-Systems

Um die Beeinflussung des Systems so gering wie eben möglich zu gestalten sollten verschiedene Maßnahmen ergriffen werden. So sollten beispielsweise benachbarte Sortieranlagen und Rückgabeautomaten in einen Mindestabstand von ca. 2 -3 m zueinander montiert werden. Die Rahmen der Anlagen sollten elektrisch isoliert sein. Gegebenenfalls sollte eine Isolation (Kunststofffolie) zwischen den Rahmen vorgesehen werden. Die Antennen selbst sollten mit einem Mindestabstand zu Metall von $10 \mathrm{~cm}$ montiert werden. Zu Gates sollte ein Mindestabstand von $8 \mathrm{~m}$ eingehalten werden.

\section{Selbstverbuchungsplätze. Mitarbeiterplätze, Rückgabeautomaten und Sortieranlagen mit UHF-Technologie}

Wie in klassischen HF-Anwendungen werden auch im UHF-Bereich an modernen Selbstverbuchungsplätzen und Mitarbeiterplätzen Lesereichweiten von maximal $30-40 \mathrm{~cm}$ benötigt. Diese können mit einem UHF-System relativ mühelos erzielt werden. Dafür eignen sich beispielsweise kleine Lesermodule, welche frei verbaut werden können oder 
fertige Desktopleser mit integrierter Antenne, welche mühelos per USB an einen PC angeschlossen werden können.
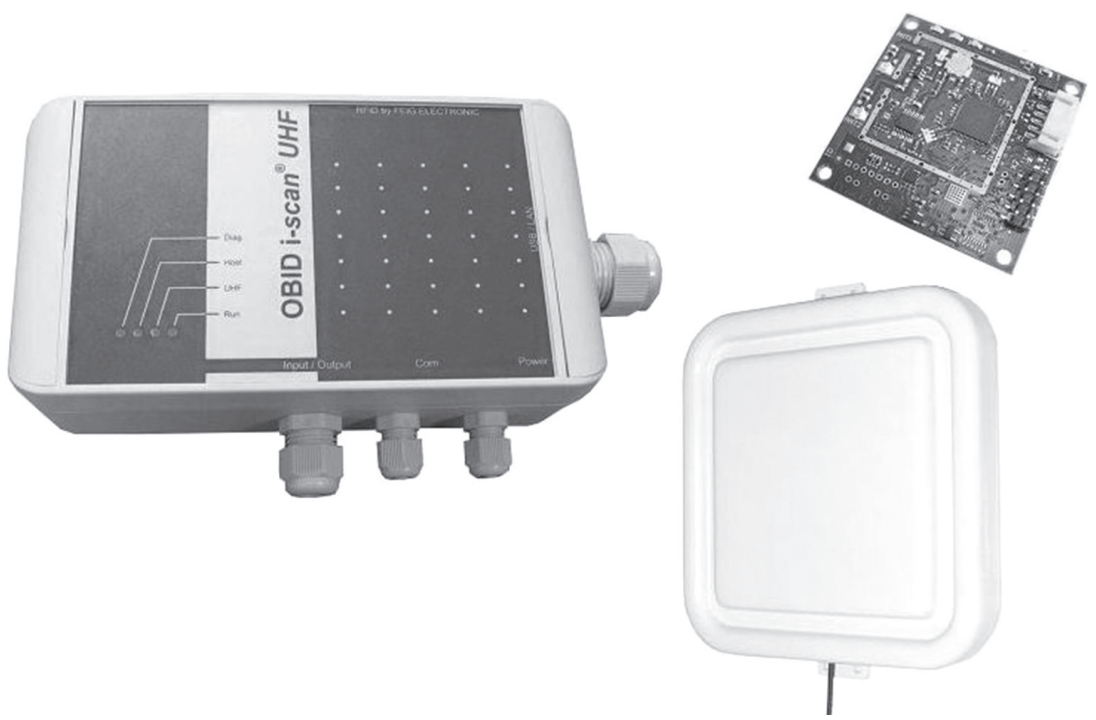

Abb. 12: UHF-Mit-Range Leser mit integrierter Antenne und UHF-Modul mit Desktopantenne

Solche Leser arbeiten gewöhnlich mit einer Leistung von wenigen Milliwatt um die benötigte Lesereichweite zu erzielen. Problematisch gestaltet sich dabei allerdings das Feld der Antenne auf den gewünschten Bereich zu beschränken. Physikalisch bedingt ist das Feld auf Grund der kleinen Bauform der Antennen weniger gerichtet. Reflektionen stellen eine zusätzliche Schwierigkeit dar. Dadurch können ebenfalls Bücher in der näheren Umgebung der Selbstverbuchungsautomaten gelesen werden. Um den Selbstverbucher herum sollten deshalb in einem Abstand von mindestens $2 \mathrm{~m}$ keine Bücher gelagert werden. Ebenso besteht das Risiko, dass Bücher, die der Kunde noch in seiner Hand hält oder Bücher, welche von wartenden oder vorbeigehenden Kunden mitgeführt werden, ebenfalls gelesen werden und zu falschen Buchungen führen. Hier sollten unbedingt weitere Abschirmmaßnahmen ergriffen werden. Am Selbstverbuchungsautomaten sollte ebenfalls der Bereich, in dem das Buch oder Medium erfasst werden sollte, seitlich und nach oben hin mit einem Absorbermaterial begrenzt werden. Dies trägt dazu bei zusätzliche Reflektionen zu vermeiden und das Lesefeld auf den gewünschten Bereich zu konzentrieren. Der Mindestabstand zwischen zwei benachbarten Arbeitsplätzen oder Selbstverbuchungsautomaten sollte ebenfalls mindestens $2 \mathrm{~m}$ betragen. Da es sich bei einem UHF-Leser um ein aktives Funksystem handelt, kann es zu einer Beeinflussung anderer elektronischer Geräte kommen. An Mitarbeiterplätzen sind davon speziell Computermonitore betroffen. Hier gilt, dass ein Mindestabstand von $1 \mathrm{~m}$ eingehalten werden sollte.

Die in einem RFID-Rückgabeautomaten vorliegenden Leseanforderungen können mit einem Mid-Range-Leser abgebildet werden. Hier besteht die Möglichkeit entweder Leser mit einer integrierten Antenne oder mir einer abgesetzten Antenne zu verwenden. Um 
eine sichere Lesung im gesamten Erfassungsbereich zu gewährleisten und Leselöcher zu schließen, empfiehlt es sich mehrere Antennen zu verbauen, welche der Reihe nach durchgeschaltet werden. Auf dem Markt sind verschiedene Leser verfügbar, welche die Möglichkeit zum Anschluss von mindestens zwei Antennen bieten. Da Rückgabeautomaten sich oftmals in einem geschlossenen Metallgehäuse befinden, sollte die Lesekammer nach allen Seiten hin mit einem Absorbermaterial ausgekleidet werden.

Zur Abbildung der Leseszenarien in Sortieranlagen können die gleichen Hardwarekomponenten verwendet werden, wie in den zuvor beschriebenen Rückgabeautomaten. $\mathrm{Zu}$ beachten ist allerdings, dass Sortieranlagen wesentlich großzügiger ausgelegt werden sollten, als es bei HF-Anwendungen der Fall ist. Grund dafür ist die wesentlich größere Lesereichweite eines UHF-Systems und mögliche Überreichweiten durch Reflektionen und die Weiterleitung von Wellen an Metall. Dies bedeutet, dass eine nahezu hundertprozentige Trennung von benachbarten Anlagen durchgeführt werden sollte. Beide Systeme sollten durch eine Schirmung voneinander getrennt werden. Ideal wäre es den Lesebereich mit Absorbermaterial in Form eines Tunnels auszukleiden. Es sollten sich keine Metallteile in der Nähe der Antenne befinden, in welches das Feld einkoppeln kann. Ebenso sollten die Rahmen der Förderanlagen, sowie alle Metallteile im gemeinsamen Bereich zweier Anlagen voneinander getrennt sein. Dadurch kann eine ungewollte Weiterleitung von Wellen vermieden werden. Zu beachten ist ebenfalls, dass nach Vereinzelung der Medien ein deutlich größerer Abstand zwischen diesen eingehalten werden muss, als es bei HF der Fall ist. Der Abstand zwischen zwei aufeinander folgenden Medien in der Förderanlage sollte $1 \mathrm{~m}$ nicht unterschreiten. Nur so kann sichergestellt werden, dass eine eindeutige Erfassung und Zuordnung der Bücher und Medien erfolgen kann. Liegen die Artikel zu dicht aneinander, so kann es zu einer zeitgleichen Erfassung der Bücher kommen, wodurch eine automatische Sortierung unmöglich wird. Ebenfalls besteht die Möglichkeit, dass ein nachfolgendes Buch vor dem vorlaufenden erkannt wird. In diesem Fall käme es zu einer falschen Sortierung der Bücher. Um das Lesefeld weitestgehend auf den gewünschten Bereich zu beschränken, empfiehlt es sich, einen Tunnel aus absorbierenden Materialien um das Band herum zu errichten. Dies erfordert allerdings einen zusätzlichen Freiraum um die Anlage herum. Durch die Verwendung von speziellen Antennen mit geringem Öffnungswinkel kann das Antennefeld weiter konzentriert und beschränkt werden. Hier ist bereits eine Vielzahl so genannter $30^{\circ}$-Antennen auf dem Markt verfügbar.

\section{Vermeidung von Stör- und Fehlerquellen bei der Installation eines HF-Systems}

Grundsätzlich arbeiten heutige RFID-Geräte bei fachgerechter Installation zuverlässig und störsicher. Trotzdem sind dies Funkanlagen, welche durch andere Sender, starke Störer oder andere Störquellen beeinflusst werden können. Typische Fehlerquellen sind:

- Schlecht abgestimmte Antennen

- »Common Mode«Störungen bzw. Ströme

- Störungen von anderen Geräten (Monitore, Antriebe, Funkgeräte) in der Nähe

- Unzureichende Lesereichweite durch zu geringe Feldstärke 
- Falsch abgestimmte oder zu kleine Transponder

- Ungewollte Lesungen

- Verringerung der Lesereichweiten durch gegenseitige Beeinflussung (Kopplung) der Transponder

- Gegenseitige Beeinflussung der RFID-Systeme durch andere in der unmittelbaren Umgebung

- Installationsfehler

\section{Schlecht abgestimmte Antennen}

Alle Antennen werden in der Regel vom Hersteller auf eine Impedanz von 50 Ohm abgestimmt. Sollten baulich bedingte Metallteile sich in der Nähe der Antenne befinden, kann ein zusätzlicher Nachgleich der Impedanz der Antenne notwendig werden. Die Impedanz kann mit Hilfe von Messgeräten überprüft werden. Ein nützliches Hilfsmittel zur Beurteilung der Anpassung der Antenne an die Impedanz von $50 \Omega$ ist das VSWR Meter. Dieses Gerät misst das Verhältnis zwischen zugeführter und reflektierter Energie. Dabei gilt ein VSWR bis zu 1.3: 1 als guter Wert. Zur Messung wird die Antenne direkt über das Antennenkabel an ein SWR Meter angeschlossen.

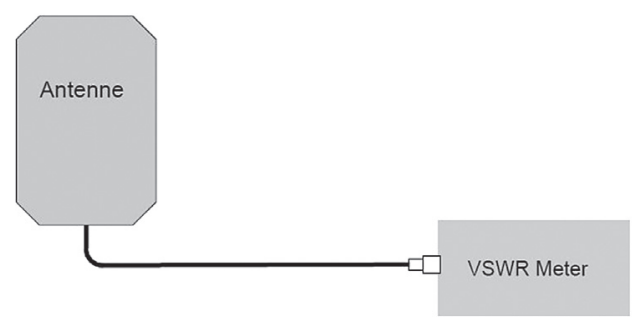

Abb. 13: Messung des SWR einer Antenne

\section{Common Mode Störungen}

Als Common Mode Störungen bezeichnet man Störungen von anderen elektrischen/ elektronischen Geräten über das Erdpotenzial. Um diese zu vermeiden, sollten unbedingt die angegebenen Mindestabstände zum Metall beachtet werden. Metallteile in der Nähe der Antennen sollten gegebenenfalls sternförmig geerdet werden. Da es sich dabei um hochfrequente Störungen handeln kann, bietet es sich an dafür die Schirmung eines separaten Antennenkabels zu verwenden.

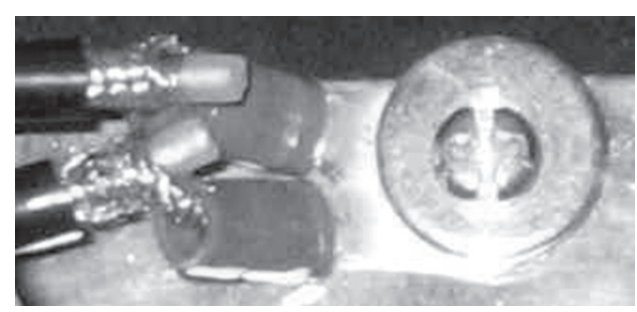

Abb. 14: Erdung über den Schirm eines zusätzlichen Antennenkabels 
Bei der Installation sollten bereits im Voraus die Vorgaben des Herstellers und Hinweise in der Montageanleitung beachtet und in der Planung mit berücksichtigt werden. Durch die Montage von zusätzlichen Ferritringen an den Kabeln können Störungen ebenfalls um ein Vielfaches reduziert und unterdrückt werden. Um die Störanfälligkeit so gering wie nur eben möglich zu halten, sollten HF-Kabel und Energieleitungen in getrennten Rohren verlegt werden.

\section{Störungen durch andere Geräte in der Nähe}

Um Störungen durch andere Geräte in der Nähe der Installation zu vermeiden, sollten unbedingt alle angegebenen Mindestabstände beachtet werden. Dies gilt im Besonderen für Energieleitungen mit hoher elektrischer Leistung. Hier darf ein Mindestabstand von $1 \mathrm{~m}$ nicht unterschritten werden. Ebenfalls können so auftretende Probleme durch die Verwendung einer zusätzlichen Schirmung und die Verlegung von Kabeln in getrennten Kabelrohren deutlich minimiert werden.

\section{Unzureichende Lesereichweite durch zu geringe Feldstärke}

Von entscheidender Bedeutung in diesem Fall sind die Eigenschaften des Lesers, der Antenne und des Transponders. Nur wenn diese optimal zueinander ausgewählt wurden, kann die bestmögliche Performance erzielt werden. Die angegeben maximalen Lesereichweiten des Herstellers sind zu beachten. In Abhängigkeit von der Größe und der Aktivierungsfeldstärke der ausgewählten Transponder müssen diese gegebenenfalls korrigiert werden. Um eine sichere Erfassung der Transponder in allen Fällen zu gewährleisten, sollten zur Sicherheit 10\% von der angegebenen Lesereichweite abgezogen werden.

\section{Falsch abgestimmte oder zu kleine Transponder}

Transponder, Antenne und Leser bilden eine Einheit. Für mittlere und große Reichweiten müssen die Transponder eine minimale Größe haben. Die Empfindlichkeit der Transponder variiert in Abhängigkeit mit der Qualität. Es ist zu beachten, dass der Transponder durch das Einbringen in einem Buch oder auf einem Medium verstimmt wird. Unter Umständen kann dieser danach nicht mehr die gleiche Performance liefern, wie es zuvor der Fall war. Bei der Bestellung der Transponder sollte deshalb dem Labelhersteller der spätere Verwendungszweck mitgeteilt werden. Mittlerweile ist eine Vielzahl verschiedener Transponder auf dem Markt verfügbar, welche speziell für die Montage auf unterschiedlichen Materialien entwickelt wurden und erst die volle Performance in Kombination mit einem bestimmten Untergrund liefern. Tests mit mehreren der verwendeten Transpondern im Voraus sind sinnvoll.

\section{Verringerung der Lesereichweite durch gegenseitige Beeinflussung (Kopplung) der Transponder}

Um eine gegenseitige Beeinflussung der Transponder untereinander zu vermeiden, sollte zwischen diesen ein Mindestabstand eingehalten werden, der in etwa der Kantenlänge 
bzw. dem Durchmesser des Transponders entspricht. Bei geringeren Abständen (z. B. dünne Büchern, CDs) ist mit einer wesentlich geringeren Empfindlichkeit zu rechnen. Hier müssen die Medien entweder vereinzelt oder RFID-Systeme mit höherer Leistung und Empfindlichkeit Verwendung finden.

\section{Ungewollte Lesungen}

Alle RFID-Antennen können Transponder in verschieden Ausrichtungen und Positionen zu der Antenne (z. B. über und unter der Antennenfläche) lesen. Treten ungewollte Lesungen auf, z. B. außerhalb eines Gates, so sollte die Anordnung der Antennen überprüft werden. Ebenso ist es möglich mittels einer zusätzlichen Schirmung den Lesebereich klar zu definieren. Unter Umständen kann mittels einer Reduktion der Sendeleistung der Lesebereich ebenfalls begrenzt werden. Hier sollte aber unter allen Umständen sichergestellt werden, dass die benötigte Lesereichweite in der Anwendung selbst sicher erzielt werden kann.

\section{Gegenseitige Beeinflussung der RFID-Systeme durch andere in der unmittelbaren Umgebung.}

Diese Probleme lassen sich oft durch die Einhaltung der angegebenen Mindestabstände, mit Hilfe von Multiplexern, Abschirmung der Antenne oder durch eine Synchronisation der Leser beheben.

\section{Installationsfehler}

Um installationsbedingte Fehler zu vermeiden ist es wichtig, dass alle Arbeiten in Übereinstimmung mit den nationalen Gesetzen und Bestimmungen für elektrische Anlagen sowie nach den örtlichen Vorschriften durchgeführt werden. Die Montageanleitungen der Antennen und Leser, sowie die Applikation-Notes des Herstellers sollten in jedem Fall mit berücksichtigt und darin enthaltene Hinweise umgesetzt werden. Dies gilt insbesondere für Angaben zu Mindestabständen, Kabellängen und zur Verlegung der Kabel.

\section{Vermeidung von Stör- und Fehlerquellen bei der Installation eines UHF-Systems}

Typische Fehlerquellen und Probleme, die bei der Installation eines UHF-Systems auftreten können, werden in dem folgenden Abschnitt gelistet und näher beschrieben.

- Interferenzen zwischen RFID-Systemen und anderen Funksystemen

- Beeinflussung des RFID-Systems durch andere elektronische Geräte und Felder

- Beeinflussung der RFID-Systeme untereinander bei zu starker Kopplung

- Reflektionen und Absorptionen

- Überreichweiten durch die Weiterleitung von Wellen

- Zu kleine oder schlecht abgeglichene Transponder 


\section{Interferenzen zwischen RFID-Systemen und anderen Funksystemen}

Der Standard EN 302208 reserviert in Europa ein Frequenzband von 865 -868 MHz exklusiv für RFID-Anwendungen im UHF-Bereich. Da es sich bei diesem Bereich nur um einen Ausschnitt des ISM-Bandes handelt, sind auf benachbarten Frequenzen eine Vielzahl anderer Funksysteme angesiedelt. Dadurch kann es zu einer gegenseitigen Beeinflussung der verschiedenen Anwendungen und Systeme kommen. Systeme, welche auf ähnlichen Frequenzen arbeiten, können verschiedene Messsysteme, wie sie beispielsweise zum Auslesen von Stromzählern eingesetzt werden, oder Funkthermometer sein. Ebenso betroffen sind davon sicherheitsrelevante Geräte, wie beispielsweise Brandmeldeanlangen. Befindet sich eine UHF-Antenne in der Nähe eines solchen Gerätes, so kann es zu einem Ausfall dessen, beziehungsweise zu einer Störung der Funkverbindung dieser Geräte kommen. Besonders kritisch ist dies bei sicherheitsrelevanten Systemen, wie Brandmeldeanlagen oder Alarmanlagen. Umgekehrt senden diese Geräte teilweise ein recht breitbandiges Signal, welches wiederum die Leser-Transponder-Kommunikation empfindlich stören kann. Besonders kritisch ist dies, wenn ein solches Signal die Transponderantwort überlagert. In diesem Fall ist eine Identifikation des Transponders unmöglich. Um dies auszuschließen, sollte das Spektrum im Frequenzbereich von $860 \mathrm{MHz}$ bis $870 \mathrm{MHz}$ mit Hilfe eines Spektrum-Analyzers überprüft werden. Wichtig ist hierbei die Messung zu einem Zeitpunkt durchzuführen, wenn alle möglichen Störsysteme eingeschaltet sind.

\section{Beeinflussung des UHF-Systems durch andere elektronische Geräte und Felder}

Leuchtstoffröhren, Computermonitore und andere elektronische Geräte strahlen oftmals starke Felder ab. Diese können die Funktion eines UHF-RFID-Systems erheblich beeinträchtigen. Kritisch ist dies jedoch nur, wenn die Antenne in unmittelbarer Nähe eines solchen Gerätes installiert wird. Wird beispielsweise eine Antenne neben einer Leuchtstoffröhre installiert, so kann es sein, dass, während die Leuchte eingeschaltet ist, die Lesereichweite drastisch zusammenbricht. Im Gegenzug kann aber auch eine Beeinflussung anderer Geräte durch ein RFID-System nicht ausgeschlossen werden. Dies ist beispielsweise bei Computermonitoren der Fall. Wird eine Antenne in unmittelbarer Nähe eines Monitors in Betrieb genommen, so kann es zu Verzerrungen und Flimmern des Bildschirms kommen. Grundsätzlich gilt, dass zwischen RFID-Systemen und anderen elektronischen Geräten ein Mindestabstand von $80 \mathrm{~cm}$ eingehalten werden sollte, damit gegenseitige Wechselwirkungen ausgeschlossen werden.

\section{Beeinflussung der UHF-Systeme untereinander}

Hauptursache für eine gegenseitige Beeinflussung von UHF-RFID-Systemen untereinander ist eine Übersteuerung des Empfängers eines Lesers. Dies ist der Fall, wenn ein zu starkes Signal in den Empfangszweig eines Lesers gekoppelt wird. Wie stark dieses Signal sein darf, kann nicht genau spezifiziert werden und ist abhängig von den Eigenschaften des Lesers. Genauere Auskunft kann der Hersteller des verwendeten Gerätes geben. Grundsätzlich kommt es zu einer Übersteuerung, wenn zwei Antennen von zwei 
unabhängigen Lesern direkt parallel zueinander ausgerichtet sind. Dies ist typischerweise der Fall bei zwei eng zusammenstehenden Gates. Bei solchen Installationen sollte ein Mindestabstand von zwei parallel zueinander ausgerichteten Antennen von mindestens $8 \mathrm{~m}$ nicht unterschritten werden. Um in solchen Situationen einer Übersteuerung entgegen zu wirken, sollte die Ausgangsleistung des Lesers soweit wie eben möglich reduziert werden. Ebenso kann in diesem Fall versucht werden die Returnlink Bitrate soweit wie möglich zu reduzieren. Dies bringt den Vorteil, dass die Eingangsfilter des Empfängers schmalbandiger werden und Störungen in einem etwas geringerem Maße eingekoppelt werden. Ebenfalls sollten die Leser getriggert werden. Dies kann beispielsweise durch einen Bewegungsmelder oder eine Lichtschranke erfolgen. Dadurch ist ein Leser nur dann aktiv, wenn dies unbedingt erforderlich ist. Dies hilft allerdings nicht eine gegenseitige Beeinflussung zu vermeiden. Eine Triggerung trägt lediglich dazu bei die Wahrscheinlichkeit für eine gegenseitige Beeinflussung zu reduzieren, da beide Leser gleichzeitig aktiv sein müssen.

Optimalen Erfolg versprechen jedoch eine zusätzliche Schirmung, bzw. eine Wand aus Absorbermaterialien zwischen beiden Antennen. Hierdurch wird eine Kopplung zwischen beiden Systemen nahezu vollständig unterbunden.

Sind zwei Antennen eines Lesers in paralleler Ausrichtung zueinander montiert, so bleibt dies ohne Auswirkungen. Verantwortlich hierfür ist ein im Leser integrierter Multiplexer, durch welchen die Antennen der Reihe nach geschaltet werden.

\section{Reflektionen und Absorptionen}

Reflektionen und Absorptionen sind am häufigsten auftretende Störgrößen in UHF-Applikationen. Oftmals treten Reflektionen und Absorptionen gleichzeitig auf. Wie zuvor bereits beschrieben, werden Reflektionen durch alle Gegenstände und Oberflächen in einem Gate und um ein Gate herum verursacht. Durch Reflektionen kommt es zu einer Überlagerung von Wellen, wodurch Leselöcher entstehen können. Um eine sichere dreidimensionale Erfassung in einem Gate zu ermöglichen, sollte deshalb unter allen Umständen mit mehreren Antennen gearbeitet werde. Ebenso sollte es vermieden werden Gegenstände dauerhaft im Bereich eines Gates abzustellen. Dies gilt insbesondere für metallische Gegenstände die Reflektionen begünstigen. Ebenso kritisch wie Metallteile sind Flüssigkeiten, die sich im Bereich vor den Antennen befinden. Wasser und andere Flüssigkeiten sowie Klebstoffe oder verschiedene Gummisorten besitzen die Eigenschaft Funkwellen zu absorbieren und eine Identifikation der dahinter liegenden Transponder zu verhindern.

\section{Überreichweiten durch die Weiterleitung von Wellen}

Funkwellen im UHF-Frequenzband können an der Oberfläche von großen Metallteilen aufgenommen und weitergeleitet werden. Dadurch können gegebenenfalls extreme Überreichweiten von bis zu 50m erzielt werden. Typischerweise tritt die Weiterleitung von Wellen an baulich bedingten Metallteilen auf. Die können zum Beispiel Stahlträger, 
Stahlbeton oder Heizungsrohre sein. Ebenso davon betroffen sind nachträglich installierte Metallteile, wie beispielsweise Förderanlagen oder große Maschinen. Zur Vermeidung eines solchen Phänomens sollten alle Metallteile in der Nähe einer Antenne abgeschirmt werden.

\section{Zu kleine oder schlecht abgeglichene Transponder}

Wie im HF-Bereich können dicht beieinander liegende Transponder eine Wechselwirkung aufeinander haben. Je dichter diese zusammen liegen, desto größer ist die gegenseitige Verstimmung. Auch kann eine Identifikation von Transpondern durch darüberliegende Transponder vermieden werden. Die Antennenfläche eines Transponders besteht aus Metall. Liegen mehrere Transponder dicht gedrängt übereinander, so kann es zu einer Abschirmung der unteren Transponder kommen.

Eine Verstimmung der Transponder erfolgt auch durch das Material, auf welchem sie aufgebracht werden. Liegt die Resonanzfrequenz eines Transponders unter idealen Bedingungen gewöhnlich bei $865 \mathrm{MHz}$, so kann diese durch die Aufbringung auf verschiedenen Materialien stark verschoben werden. Durchgeführte Tests haben ergeben, dass beispielsweise die Resonanzfrequenz eines Transponders nach Aufbringung in einem Buch um bis zu $200 \mathrm{MHz}$ herabgesenkt werden kann. Eine solche Verschiebung der Resonanzfrequenz hat auf die grundsätzliche Funktion eines Transponders keinen Einfluss. Hierdurch wird jedoch die maximal mögliche Performance des Transponders bei der gesetzlich vorgeschriebenen Betriebsfrequenz verringert. Um solche Phänomene zu verhindern, sind verschiedene, speziell vorverstimmte Transponder auf dem Markt verfügbar. Diese werden durch das Material, auf welchem sie aufgebracht werden, richtig gestimmt und zeigen in Kombination mit dem speziellen Untergrund erst die bestmögliche Performance.

\section{Fazit}

Stellt man die Eigenschaften der HF-Technologie und der UHF-Technologie gegenüber, so ist zuerkennen, dass beide RFID-Systeme Ihre Vor- und Nachteile besitzen.

Der große Vorteil der UHF-Technologie besteht dabei in der hohen Reichweite, welche erzielt werden kann. Problematisch ist allerdings, dass es bedingt durch Reflektionen zu Leselöchern und Überreichweiten kommen kann. Das Feld lässt sich nur sehr schwierig und aufwändig begrenzen. Um eine dreidimensionale Erfassung zu ermöglichen, muss mit mehreren Antennen gearbeitet werden.

Mit einem HF-System ist die maximale Lesereichweite deutlich geringer. Dafür besitzt ein HF-System den Vorteil, dass durch das Prinzip der induktiven Kopplung ein sehr homogenes Feld aufgebaut wird, in dem der Transponder sicher erkannt werden kann. HF-Antennen werden vom Hersteller auf eine Impedanz von 50 Ohm angepasst. Dieser Impedanzwert sollte aber nach erfolgter Installation der Antennen nochmals überprüft werden. Gegebenenfalls muss die Antenne nachgeglichen werden. Bei einem 
UHF-System erfolgt eine derartige Beeinflussung der Antenne nicht. Somit kann auf eine zusätzliche Überprüfung der Antennen verzichtet werden.

Kritisch für den Betrieb beider Systeme sind Metalle in der Nähe der Installation. Bei HF wirken sich Metalle sehr stark auf die Impedanz der Antenne aus und verstimmen diese. Nach Einhaltung der zuvor angegeben Mindestabstände und einem durchgeführten Antennenabgleich ist dieser Einfluss jedoch nahezu kompensiert und das System stabil. Im UHF-Bereich werden durch Metalle zusätzliche Reflektionen verursacht, wodurch eine sichere Erfassung des Transponders im gesamten Durchgangsbereich problematisch werden kann. Auch werden bei einem UHF-System durch kleinste Änderungen im Erfassungsbereich die Eigenschaften des Feldes stark beeinflusst. Dies können beispielsweise Personen sein, welche durch das Gate hindurchgehen oder Pflanzen, welche zur Dekoration aufgestellt werden. Mit jeder Änderung der Umgebungsbedingungen entstehen neue Reflektionen, wodurch es zu einer Verschiebung des Antennenfeldes kommen kann. Es entstehen neue Leselöcher. Dadurch lässt sich die Ausbreitung eines UHF-Feldes niemals genau vorhersagen und berechnen.

Auf Grund der Vielzahl von Erfahrungen, welche bereits mit HF-Systemen im Bibliotheksumfeld gesammelt wurden und der hohen Anzahl von erfolgreich installierten Systemen wäre die HF-RFID-Technologie nach dem jetzigen Stand der Technik für RFIDInstallationen in Bibliotheken zu bevorzugen. 